\title{
Double sampling plan for Truncated Life test based on Kumaraswamy-Log-Logistic Distribution
}

\author{
Dr. S. Muthulakshmi, B. Geetha Gnana Selvi \\ Professor in Statistics, M.Phil Scholar \\ Department of Mathematics \\ Avinashilingam Institute for Home Science and High Education for Women, Coimbatore - 641043.
}

\begin{abstract}
A double acceptance sampling plan for the life test truncated at the pre-assigned time to decide on acceptance or rejection of the submitted lots is considered. This double acceptance sampling plan uses only zero and one failure scheme. The probability model of the lifetime of the product is specified as Kumaraswamy-log-logistic distribution which contains several sub models. The minimum sample sizes of the first and second samples necessary to ensure the specified median life are obtained at the given consumer's confidence level. The operating characteristic values are analyzed with various ratios of the true median lifetime to the specified lifetime of the product. The minimum ratios of the median life to the specified life are also obtained so as to lower the producer's risk at the specified level. Numerical examples are provided to explain the application of sampling plan.
\end{abstract}

Keywords: Consumer's confidence level; double sampling plan; Kumaraswamy-log-logistic distribution; Median life; Poisson distribution.

\section{Introduction}

Products or items have variations in their lifetimes even though they are produced by the same producer, machine and under the same manufacturing conditions. The producer and the consumer are subject to risks due to the decision on the acceptance or rejection of products based on an acceptance sampling plan. Consumer's risk is due to the acceptance of lots with several products having the median lifetime less than the specified lifetime and the producer's risk is due to the rejection of the lots with several products having median lifetime greater than the specified lifetime. Increasing the sample size may minimize both risks to certain level but this will obviously increase the cost of inspection. Thus an efficient acceptance sampling scheme and truncated life tests are the only option to reduce these risks.

The design parameters of a sampling plan depend on the underlying statistical distribution. Several studies have been done for designing single sampling plans based on the truncated life tests under various statistical distributions. Epstein [4] first introduced single acceptance sampling plans for the truncated life test based on the exponential distribution. Goode and Kao [5] developed an acceptance sampling plan using the Weibull distribution as a lifetime distribution. Gupta and Groll [7] derived the acceptance sampling plan for the gamma distribution and Gupta [6] designed the plan for the lifetime of the product having log-normal distribution.

Duncan [3] pointed out that double sampling plans reduce the sample size or producer's risk in the area of quality control. Aslam and Jun [1] introduced double acceptance sampling for the truncated life test based on the generalized log-logistic distribution. The purpose of this paper is to propose the double acceptance sampling plan for the truncated life test assuming that the lifetime of a product follows the Kumaraswamy-log-logistic distribution. The beauty and importance of this distribution lies in accommodating several importance distributions as sub-models. The minimum sample sizes of the first and second samples for zero-one double sampling plan are determined at the specified consumer's confidence level. The operating characteristics are analyzed as a function of the ratio of the true median life to the specified life. The Kumaraswamy-log-logistic distribution is introduced in Section 2. The proposed double sampling plan is given in Section 3. The operating characteristics are analyzed in Section 4. The minimum median ratios to the specified life are presented in Section 5. Numerical examples for selection of plans for a given condition are presented in Section 6.

\section{Kumaraswamy-Log-Logistic Distribution}

The lifetime of a product is assumed to have the Kumaraswamy-log-logistic distribution (KumLL), whose probability density function (pdf) and cumulative distribution function (cdf) are given respectively as

$f(t)=\frac{a b \gamma}{\alpha^{a \gamma}} t^{a \gamma-1}\left[1+\left(\frac{t}{\alpha}\right)^{\gamma}\right]^{-(a+1)}\left\{1-\left[1-\frac{1}{1+\left(\frac{t}{\alpha}\right)^{\gamma}}\right]^{a}\right\}^{b-1}, \quad \mathrm{t}>0, \mathrm{a}, \mathrm{b}, \alpha$ and $\gamma>0$ 
and

$$
F(t ; a, b, \alpha, \gamma)=1-\left\{1-\left[1-\frac{1}{1+\left(\frac{t}{\alpha}\right)^{\gamma}}\right]^{a}\right\}^{b}, \quad \mathrm{t} \geq 0, \mathrm{a}, \mathrm{b}, \alpha \text { and } \gamma>0
$$

where $\alpha$ is a scale parameter and $\mathrm{a}, \mathrm{b}$ and $\gamma$ are the shape parameters. Four parameter Kumaraswamy-log-logistic distribution is the result of the work done by Kumaraswamy [8] and Cordeiro and de Castro [2]. If T is a random variable relating to lifetime of the product with density function (1) is denoted as $\mathrm{T} \sim \operatorname{KumLL}(\mathrm{a}, \mathrm{b}, \alpha, \gamma)$. The median of the Kumaraswamy-log-logistic distribution is derived by

$$
m=\alpha\left[\frac{\left\{1-(0.5)^{\frac{1}{b}}\right\}^{\frac{1}{a}}}{1-\left\{1-(0.5)^{\frac{1}{b}}\right\}^{\frac{1}{a}}}\right]^{\frac{1}{\gamma}}
$$

Expression (3) shows that the median is proportional to the scale parameter, $\alpha$ when the two shape parameters $a$ and $b$ are fixed. It is also seen that the median reduces to $m=\alpha$ regardless of $\gamma$ with $a=b=1$ for the Kumaraswamy-log-logistic distribution.

Kumaraswamy-log-logistic distribution reduces to Burr distribution when $a=1$, exponentiated $\log$ - $\log$ istic distribution when $\mathrm{b}=1$ and $\log$-logistic distribution when $\mathrm{a}=\mathrm{b}=1$.

\section{Design Of The Double Sampling Plan}

Assume that the quality of a product can be represented by its median lifetime, $\mathrm{m}$. The lot will be accepted if the submitted lot has a good quality when the experimental data supports the null hypothesis, $\mathrm{H}_{0}$ : $\mathrm{m} \geq \mathrm{m}_{0}$ against the alternative hypothesis, $\mathrm{H}_{1}: \mathrm{m}<\mathrm{m}_{0}$. where $\mathrm{m}_{0}$ is a specified lifetime. The significance level for the test is used through 1- $\mathrm{P}^{*}$, where $\mathrm{P}^{*}$ is the consumer's confidence level.

The double sampling plan for the truncated life test consists of (i) the number of units of first and second samples (ii) acceptance numbers (iii) the ratio of true median life to the specified median life $\mathrm{m} / \mathrm{m}_{0}$. The consumer risk, the probability of accepting a bad lot which has the true median life below the specified life $\mathrm{m}_{0}$, is fixed as not to exceed 1-P*. The operating procedure of zero-one double sampling plan for the truncated life test has the following steps

1. Draw a first random sample of size $\mathrm{n}_{1}$ from the submitted lot and put on the test for pre-assigned experimental time $t_{0}$. If there are $c_{1}$ or less failures occur within the experimental time accept the lot. If there are $c_{2}+1$ failures occur by $t_{0}$ reject the lot.

2. If the number of failures by $t_{0}$ is between $c_{1}+1$ and $c_{2}$ then draw a second sample of size $n_{2}$ and put them on the test for time $t_{0}$. If the number of failures in combined sample is at most $c_{2}$ accept the lot. Otherwise, reject the lot.

Double sampling plan with $\mathrm{c}_{1}=0$ and $\mathrm{c}_{2}=1$ is the zero-one double sampling plan. Often it is convenient to set the termination time as a multiple of the specified lifetime $\mathrm{m}_{0}$, in which case $\mathrm{t}_{0}=\eta \mathrm{m}_{0}$ for a specified multiplier $\eta$. Then the proposed sampling plan is characterized by five parameters $\left(n_{1}, n_{2}, c_{1}, c_{2}, \eta\right)$, where $c_{1}<c_{2}$.

It is assumed that the lot size is large enough to use the Poisson distribution to calculate the probability of acceptance of the lot. Under the proposed double acceptance sampling plan the probability of acceptance of the lot is

$$
P_{a}=\sum_{i=0}^{c_{1}} \frac{e^{-n_{1} p}\left(n_{1} p\right)^{i}}{i !}+\sum_{x=c_{1}+1}^{c_{2}} \frac{e^{-n_{1} p}\left(n_{1} p\right)^{x}}{x !}\left[\sum_{i=0}^{c_{2}-x} \frac{e^{-n_{2} p}\left(n_{2} p\right)^{i}}{i !}\right]
$$

where $\mathrm{p}$, the probability that an item fails before $\mathrm{t}_{0}$ is given by

$$
p=1-\left\{1-\left(1-\frac{1}{1+\left(\frac{t_{0}}{\alpha}\right)^{\gamma}}\right)^{a}\right\}^{b}
$$




$$
=1-\left\{1-\left(\frac{(\eta \delta)^{\gamma}}{\left(\frac{m}{m_{0}}\right)^{\gamma}+(\eta \delta)^{\gamma}}\right)^{a}\right\}^{b}
$$

and

$$
\delta=\left[\frac{\left\{1-(0.5)^{\frac{1}{b}}\right\}^{\frac{1}{a}}}{1-\left\{1-(0.5)^{\frac{1}{b}}\right\}^{\frac{1}{a}}}\right]^{\frac{1}{\gamma}}
$$

The proposed double sampling plan having $c_{1}=0$ and $c_{2}=1$ is called as zero and one failure double sampling scheme. Therefore, the probability of acceptance of the lot is

$$
P_{a}=e^{-n_{1} p}\left\{1+e^{-n_{2} p}\left(n_{1} p\right)\right\}
$$

The minimum sample sizes $\mathrm{n}_{1}$ and $\mathrm{n}_{2}$ ensuring $\mathrm{m} \geq \mathrm{m}_{0}$ at the consumer's confidence level $\mathrm{P}^{*}$ can be found as the solution of

$$
e^{-n_{1} p_{0}}\left\{1+e^{-n_{2} p_{0}}\left(n_{1} p_{0}\right)\right\} \leq 1-P^{*}
$$

where $\mathrm{p}_{0}$ is the value of $\mathrm{p}$ evaluated at $\mathrm{m}=\mathrm{m}_{0}$ as

$$
p_{0}=1-\left\{1-\left(\frac{(\eta \delta)^{\gamma}}{1+(\eta \delta)^{\gamma}}\right)^{a}\right\}^{b}
$$

Equation (7) provides multiple solutions for sample sizes $n_{1}$ and $n_{2}$ satisfying the specified confidence level. In order to find the optimal sample sizes the minimum of ASN is incorporated along with the probability of the acceptance of the lot $\leq 1-\mathrm{P}^{*}$ and $\mathrm{n}_{2} \leq \mathrm{n}_{1}$.

The ASN for double sampling plan is

$\mathrm{ASN}=n_{1} P_{1}+\left(n_{1}+n_{2}\right)\left(1-P_{1}\right)$

where $P_{1}$ is the probability of acceptance or rejection based on the first sample that is given by

$$
P_{1}=1-\sum_{i=c_{1}+1}^{c_{2}} \frac{e^{-n_{1} p}\left(n_{1} p\right)^{i}}{i !}
$$

For $\mathrm{c}_{1}=0$ and $\mathrm{c}_{2}=1$, we have

$$
\operatorname{ASN}=n_{1}+n_{1} n_{2} p e^{-n_{1} p}
$$

Therefore, evaluation of minimum sample sizes reduces to the following optimization problem.

Minimize

$$
\mathrm{ASN}=n_{1}+n_{1} n_{2} p_{0} e^{-n_{1} p_{0}}
$$

subject to

$$
P_{a}=e^{-n_{1} p_{0}}\left\{1+e^{-n_{2} p_{0}}\left(n_{1} p_{0}\right)\right\} \leq 1-P^{*}
$$

where $\mathrm{n}_{1}$ and $\mathrm{n}_{2}$ are integers with $1 \leq \mathrm{n}_{2} \leq \mathrm{n}_{1}$

Minimum sample sizes may be obtained from (11) by a simple search by varying the values of $n_{1}$ and $n_{2}$. Table 1 presents the minimum sample sizes for the first and second samples when the lifetime of the product follows the Kumaraswamy-log-logistic distribution for various values of $\mathrm{P}^{*}, \eta$ and different combinations of $(\mathrm{a}, \mathrm{b}, \gamma)$. Numerical results in Table 1 reveal that

i. increase in the shape parameters $(a, b, \gamma)$ increases the sample sizes rapidly for given $\eta$ at the specified confidence level.

ii. increase in $\eta$ decreases the minimum sample sizes at a given confidence level irrespective of $(a, b, \gamma)$ and iii. increase in confidence level increases the minimum sample sizes for a specified $\eta$ irrespective of $(a, b, \gamma)$.

Table 1. Minimum sample sizes to assure the median life to exceed a given value $\mathrm{m}_{0}$ for various $(\mathrm{a}, \mathrm{b}, \gamma)$

\begin{tabular}{lllllllllll}
\hline & & \multicolumn{10}{c}{$\eta$} \\
\cline { 3 - 10 }$(\mathrm{a}, \mathrm{b}, \gamma)$ & $\mathrm{P}^{*}$ & 0.3 & 0.5 & 0.7 & 0.9 & 1.1 & 1.3 & 1.5 & 1.7 & 1.9 \\
\hline$(1,1,2)$ & 0.75 & 21,18 & 9,7 & 6,3 & 4,3 & 4,2 & 3,2 & 3,2 & 3,1 & 3,1 \\
& 0.90 & 31,27 & 13,11 & 8,6 & 6,4 & 5,3 & 4,4 & 4,3 & 4,2 & 4,2 \\
& 0.95 & 38,37 & 16,14 & 10,7 & 7,7 & 6,5 & 5,5 & 5,3 & 5,2 & 4,4
\end{tabular}




\begin{tabular}{|c|c|c|c|c|c|c|c|c|c|c|}
\hline & 0.99 & 57,46 & 24,16 & 15,8 & 11,6 & 9,5 & 8,4 & 7,5 & 7,3 & 7,2 \\
\hline \multirow[t]{4}{*}{$(1,1,4)$} & 0.75 & 207,206 & 29,26 & 9,8 & 5,3 & 3,3 & 3,1 & 2,2 & 2,2 & 2,2 \\
\hline & 0.90 & 310,310 & 43,40 & 13,13 & 7,4 & 5,2 & 4,2 & 3,3 & 3,2 & 3,2 \\
\hline & 0.95 & 390,381 & 54,48 & 17,12 & 8,8 & 6,3 & 5,2 & 4,3 & 4,2 & 4,2 \\
\hline & 0.99 & 579,569 & 80,65 & 25,16 & 12,9 & 8,6 & 7,3 & 6,3 & 6,2 & 6,2 \\
\hline \multirow[t]{4}{*}{$(1,2,2)$} & 0.75 & 24,22 & 10,8 & 6,4 & 4,4 & 3,3 & 3,2 & 3,1 & 3,1 & 2,2 \\
\hline & 0.90 & 36,32 & 14,14 & 9,5 & 6,5 & 5,3 & 4,3 & 4,2 & 4,2 & 3,3 \\
\hline & 0.95 & 45,40 & 18,15 & 11,7 & 8,4 & 6,4 & 5,4 & 5,2 & 4,4 & 4,3 \\
\hline & 0.99 & 66,63 & 26,26 & 16,9 & 11,7 & 9,5 & 8,3 & 7,3 & 6,5 & 6,3 \\
\hline \multirow[t]{4}{*}{$(1,2,4)$} & 0.75 & 249,248 & 34,32 & 10,9 & 5,3 & 3,2 & 3,1 & 2,2 & 2,2 & 2,1 \\
\hline & 0.90 & 373,373 & 50,50 & 15,12 & 7,5 & 5,2 & 4,2 & 3,2 & 3,2 & 3,2 \\
\hline & 0.95 & 468,468 & 63,61 & 19,14 & 9,5 & 6,3 & 4,4 & 4,2 & 4,2 & 4,1 \\
\hline & 0.99 & 697,683 & 94,82 & 27,26 & 13,7 & 8,5 & 6,5 & 6,2 & 5,4 & 5,3 \\
\hline \multirow[t]{4}{*}{$(2,2,4)$} & 0.75 & 9270,9270 & 177,175 & 18,16 & 5,5 & 3,2 & 2,2 & 2,2 & 2,1 & 2,1 \\
\hline & 0.90 & 13902,13900 & 265,264 & 27,23 & 8,5 & 4,4 & 3,3 & 3,2 & 3,2 & 3,2 \\
\hline & 0.95 & 17443,17435 & 333,326 & 33,32 & 10,6 & 5,5 & 4,3 & 4,2 & 4,2 & 4,1 \\
\hline & 0.99 & 25952,25939 & 495,478 & 49,47 & 14,9 & 8,4 & 6,3 & 6,2 & 5,3 & 5,3 \\
\hline \multirow[t]{4}{*}{$(2,3,4)$} & 0.75 & 12348,12347 & 228,224 & 21,19 & 6,3 & 3,2 & 2,2 & 2,2 & 2,1 & 2,1 \\
\hline & 0.90 & 18518,18512 & 341,338 & 31,30 & 8,6 & 4,3 & 3,3 & 3,2 & 3,2 & 3,2 \\
\hline & 0.95 & 23233,23232 & 428,421 & 39,36 & 10,7 & 5,4 & 4,2 & 4,2 & 4,1 & 4,1 \\
\hline & 0.99 & 34568,34554 & 636,625 & 58,49 & 15,8 & 8,3 & 6,3 & 5,4 & 5,3 & 5,3 \\
\hline
\end{tabular}

The effect of the change in the shape parameter a when $b=2$ and $\gamma=4$ with reference to the minimum first sample size and the experiment time when the confidence level is fixed at 0.90 for Kumaraswamy-log-logistic distribution is given in Fig.1.

Fig.1. The first sample size vs. Experiment time at confidence level, $\mathrm{P}^{*}=0.90$ for $(\mathrm{a}, 2,4)$

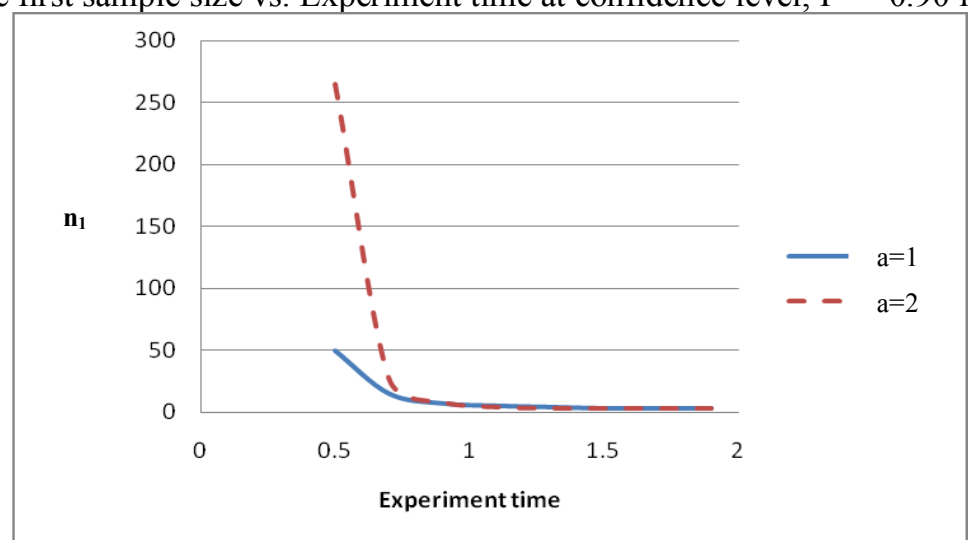

The figure indicates that increase in the shape parameter a increases the first sample size rapidly for shorter experimenter time compared to the increase of the sample size for longer experimental time.

Fig 2 shows the trend of first sample sizes against the test time for the effect of the change in the shape parameter $\mathrm{b}$ when $\mathrm{a}=1$ and $\gamma=4$ at the confidence level, $\mathrm{P}^{*}=0.90$. This figure indicates that at the specified confidence level experimental time of longer duration requires lesser sample sizes compared to experimental time of shorter duration.

Fig.2. The first sample size vs. Experiment time at confidence level, $\mathrm{P}^{*}=0.90$ for $(1, \mathrm{~b}, 4)$ 


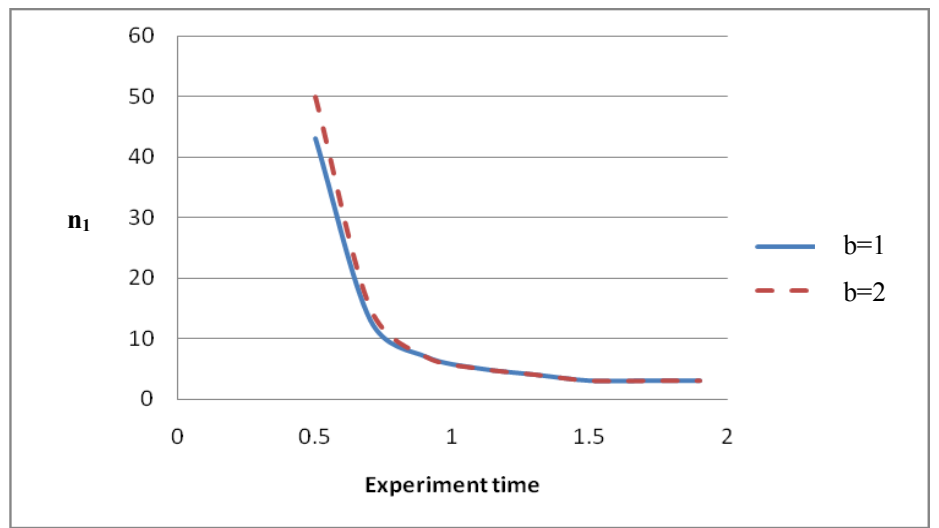

The effect of the change in the shape parameter $\gamma$ when $a=1$ and $b=2$ with reference to the minimum first sample size and the experiment time when the confidence level is fixed at 0.90 for Kumaraswamy-log-logistic distribution is given in Fig.3.

Fig.3. The first sample size vs. Experiment time at confidence level, $\mathrm{P}^{*}=0.90$ for $(1,2, \gamma)$

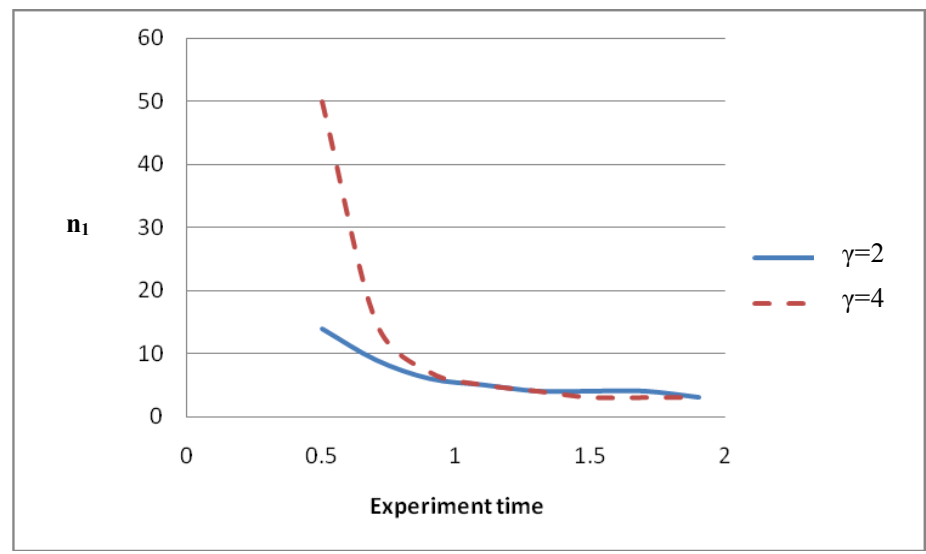

The figure indicates that increase in the shape parameter increases the first sample size rapidly for shorter experiment time compared to the sample size for longer experimental time.

\section{Operating Characteristic Values}

OC values depict the performance of the sampling plan according to the submitted quality of the product. The probability of the acceptance will increase if the true median increases beyond the specified life. Therefore, we need to know the operating characteristic values for the proposed plan according to the ratio $\mathrm{m} / \mathrm{m}_{0}$ of the true median life to the specified life. Obviously, a plan will be more desirable if its $\mathrm{OC}$ values increase more sharply to one. The OC values as a function of the ratio $\mathrm{m} / \mathrm{m}_{0}$ when $\mathrm{P}^{*}=0.90, \mathrm{a}=1, \mathrm{~b}=2, \gamma=4$ and $\eta=0.7$ are presented in Table 2. It is seen that the OC increases to one more rapidly to move from a lower value to a higher value of the ratio $\mathrm{m} / \mathrm{m}_{0}$. This arises due to the increase in the sample sizes required at higher shape parameters. It is also seen that i. increase in confidence level decreases the operating characteristic values for a given ratio $\mathrm{m} / \mathrm{m}_{0}$ ii. increase in confidence level decreases the operating characteristic values for a given $\eta$ iii. increase in the ratio $\mathrm{m} / \mathrm{m}_{0}$ increases the operating characteristic values for any given $\eta$ and any specified confidence level

Table 2. OC values with $\mathrm{a}=1, \mathrm{~b}=2$ and $\gamma=4$ at specified consumer's confidence level

\begin{tabular}{|c|c|c|c|c|c|c|c|c|c|}
\hline \multirow[b]{2}{*}{$\mathrm{P} *$} & \multirow[b]{2}{*}{$\mathrm{n}_{1}$} & \multirow[b]{2}{*}{$\mathrm{n}_{2}$} & \multirow[b]{2}{*}{$\eta$} & \multicolumn{5}{|c|}{$\mathrm{m} / \mathrm{m} 0$} & \multirow[b]{2}{*}{12} \\
\hline & & & & 2 & 4 & 6 & 8 & 10 & \\
\hline \multirow[t]{3}{*}{0.75} & 249 & 248 & 0.3 & 0.985628 & 0.999937 & 0.999998 & 1 & 1 & 1 \\
\hline & 34 & 32 & 0.5 & 0.984747 & 0.999932 & 0.999997 & 1 & 1 & 1 \\
\hline & 10 & 9 & 0.7 & 0.981584 & 0.999916 & 0.999997 & 1 & 1 & 1 \\
\hline
\end{tabular}




\begin{tabular}{|c|c|c|c|c|c|c|c|c|c|}
\hline & 5 & 3 & 0.9 & 0.974433 & 0.999878 & 0.999995 & 1 & 1 & 1 \\
\hline & 3 & 2 & 1.1 & 0.956752 & 0.999769 & 0.999991 & 0.999999 & 1 & 1 \\
\hline & 3 & 1 & 1.3 & 0.905425 & 0.999383 & 0.999975 & 0.999998 & 1 & 1 \\
\hline & 2 & 2 & 1.5 & 0.828536 & 0.998489 & 0.999938 & 0.999994 & 0.999999 & 1 \\
\hline & 2 & 2 & 1.7 & 0.700451 & 0.996053 & 0.999833 & 0.999983 & 0.999997 & 0.999999 \\
\hline & 2 & 1 & 1.9 & 0.648963 & 0.993818 & 0.99973 & 0.999972 & 0.999995 & 0.999999 \\
\hline 0.90 & 373 & 373 & 0.3 & 0.969613 & 0.999858 & 0.999994 & 0.999999 & 1 & 1 \\
\hline & 50 & 50 & 0.5 & 0.967821 & 0.999849 & 0.999994 & 0.999999 & 1 & 1 \\
\hline & 15 & 12 & 0.7 & 0.963795 & 0.999826 & 0.999993 & 0.999999 & 1 & 1 \\
\hline & 7 & 5 & 0.9 & 0.948866 & 0.999737 & 0.99999 & 0.999999 & 1 & 1 \\
\hline & 5 & 2 & 1.1 & 0.915744 & 0.999509 & 0.99998 & 0.999998 & 1 & 1 \\
\hline & 4 & 2 & 1.3 & 0.827034 & 0.998698 & 0.999947 & 0.999995 & 0.999999 & 1 \\
\hline & 3 & 2 & 1.5 & 0.739319 & 0.997386 & 0.999892 & 0.999989 & 0.999998 & 1 \\
\hline & 3 & 2 & 1.7 & 0.570748 & 0.993217 & 0.999708 & 0.99997 & 0.999995 & 0.999999 \\
\hline & 3 & 2 & 1.9 & 0.412318 & 0.984554 & 0.999299 & 0.999928 & 0.999988 & 0.999997 \\
\hline 0.95 & 468 & 468 & 0.3 & 0.954362 & 0.999778 & 0.999991 & 0.999999 & 1 & 1 \\
\hline & 63 & 61 & 0.5 & 0.952258 & 0.999765 & 0.999991 & 0.999999 & 1 & 1 \\
\hline & 19 & 14 & 0.7 & 0.947206 & 0.999735 & 0.99999 & 0.999999 & 1 & 1 \\
\hline & 9 & 5 & 0.9 & 0.929708 & 0.999623 & 0.999985 & 0.999998 & 1 & 1 \\
\hline & 6 & 3 & 1.1 & 0.876009 & 0.999219 & 0.999969 & 0.999997 & 0.999999 & 1 \\
\hline & 4 & 4 & 1.3 & 0.77111 & 0.998066 & 0.999921 & 0.999992 & 0.999999 & 1 \\
\hline & 4 & 2 & 1.5 & 0.653227 & 0.996061 & 0.999835 & 0.999983 & 0.999997 & 0.999999 \\
\hline & 4 & 2 & 1.7 & 0.459191 & 0.98985 & 0.999557 & 0.999955 & 0.999992 & 0.999998 \\
\hline & 4 & 1 & 1.9 & 0.365964 & 0.982445 & 0.9992 & 0.999918 & 0.999986 & 0.999997 \\
\hline 0.99 & 697 & 683 & 0.3 & 0.91045 & 0.999517 & 0.999981 & 0.999998 & 1 & 1 \\
\hline & 94 & 82 & 0.5 & 0.910191 & 0.999515 & 0.999981 & 0.999998 & 1 & 1 \\
\hline & 27 & 26 & 0.7 & 0.890217 & 0.999373 & 0.999975 & 0.999997 & 1 & 1 \\
\hline & 13 & 7 & 0.9 & 0.87212 & 0.999232 & 0.999969 & 0.999997 & 0.999999 & 1 \\
\hline & 8 & 5 & 1.1 & 0.789316 & 0.998456 & 0.999938 & 0.999994 & 0.999999 & 1 \\
\hline & 6 & 5 & 1.3 & 0.635139 & 0.9962 & 0.999842 & 0.999984 & 0.999997 & 0.999999 \\
\hline & 6 & 2 & 1.5 & 0.498256 & 0.992774 & 0.999693 & 0.999969 & 0.999995 & 0.999999 \\
\hline & 5 & 4 & 1.7 & 0.285686 & 0.980302 & 0.999108 & 0.999908 & 0.999985 & 0.999996 \\
\hline & 5 & 3 & 1.9 & 0.175091 & 0.962528 & 0.998192 & 0.999812 & 0.999968 & 0.999993 \\
\hline
\end{tabular}

\section{Minimum Median Ratios To The Specified Life}

Consider now that the producer wants to know what will be the minimum product quality level in order to keep the producer's risk at the specified level. At the producer's risk of $\alpha$ the minimum ratio $\mathrm{m} / \mathrm{m}_{0}$ can be obtained by solving

$$
P_{a} \geq 1-\alpha
$$

where $\mathrm{P}_{\mathrm{a}}$ is given by Equation (4) or (6) for the zero and one failure schemes and $\mathrm{p}$ is given by Equation (5). Tables 3 and 4 show the minimum median ratios to the specified life at the given consumer's confidence level and test times corresponding to the producer's risks of 0.05 and 0.10 .

Numerical values in tables reveal that

i. increase in producer's risk decreases the minimum median ratios for any given combinations of $(a, b, \gamma), \eta$ and $\mathrm{P}^{*}$ 
ii. increase in $\mathrm{P}^{*}$ increases the minimum median ratios for any given combinations of $(\mathrm{a}, \mathrm{b}, \gamma)$ and $\eta$ for a given producer's risk

iii. increase in $\eta$ increases the minimum median ratios for any given combinations of $(\mathrm{a}, \mathrm{b}, \gamma)$ and $\mathrm{P}^{*}$ for a given producer's risk

Table 3. Minimum median ratios to the specified life at producer's risk of 0.05

\begin{tabular}{|c|c|c|c|c|c|c|c|c|c|c|}
\hline \multirow[b]{2}{*}{$(a, b, \gamma)$} & \multirow[b]{2}{*}{$\mathrm{P}^{*}$} & \multicolumn{7}{|c|}{$\eta$} & \multirow[b]{2}{*}{1.7} & \multirow[b]{2}{*}{1.9} \\
\hline & & 0.3 & 0.5 & 0.7 & 0.9 & 1.1 & 1.3 & 1.5 & & \\
\hline \multirow[t]{4}{*}{$(1,1,2)$} & 0.75 & 2.9368 & 3.1345 & 3.3447 & 3.6802 & 4.2442 & 4.4752 & 5.1637 & 5.3461 & 5.9751 \\
\hline & 0.90 & 3.5830 & 3.8309 & 4.1081 & 4.4729 & 4.8958 & 5.5698 & 6.1335 & 6.5591 & 7.3307 \\
\hline & 0.95 & 4.0397 & 4.2790 & 4.5595 & 5.1602 & 5.6548 & 6.2610 & 6.6761 & 7.1829 & 8.1404 \\
\hline & 0.99 & 4.8098 & 5.0448 & 5.4014 & 5.9463 & 6.5730 & 7.2115 & 8.1574 & 8.6356 & 9.2453 \\
\hline \multirow[t]{4}{*}{$(1,1,4)$} & 0.75 & 1.6867 & 1.7030 & 1.7709 & 1.8988 & 2.1092 & 2.3054 & 2.5746 & 2.9178 & 3.2611 \\
\hline & 0.90 & 1.8668 & 1.8858 & 1.9636 & 2.0659 & 2.2611 & 2.5536 & 2.8762 & 3.1542 & 3.5252 \\
\hline & 0.95 & 1.9734 & 1.9898 & 2.0462 & 2.2304 & 2.4045 & 2.6722 & 3.0332 & 3.3393 & 3.7321 \\
\hline & 0.99 & 2.1794 & 2.1809 & 2.2402 & 2.4187 & 2.6648 & 2.9300 & 3.2788 & 3.6296 & 4.0566 \\
\hline \multirow[t]{4}{*}{$(1,2,2)$} & 0.75 & 2.8927 & 3.0325 & 3.1823 & 3.5332 & 3.7143 & 4.1150 & 4.3460 & 4.9255 & 5.1653 \\
\hline & 0.90 & 3.5297 & 3.7250 & 3.8204 & 4.2308 & 4.4838 & 4.8737 & 5.3109 & 6.0190 & 6.4156 \\
\hline & 0.95 & 3.9481 & 4.1092 & 4.3101 & 4.5624 & 5.0007 & 5.5276 & 5.8083 & 6.6737 & 7.1231 \\
\hline & 0.99 & 4.8380 & 5.0894 & 5.1254 & 5.5415 & 6.0034 & 6.3714 & 6.9685 & 7.9914 & 8.3074 \\
\hline \multirow[t]{4}{*}{$(1,2,4)$} & 0.75 & 1.6855 & 1.6983 & 1.7398 & 1.8171 & 1.9571 & 2.2128 & 2.4733 & 2.8030 & 2.9677 \\
\hline & 0.90 & 1.8654 & 1.8799 & 1.9105 & 2.0066 & 2.1646 & 2.4462 & 2.6688 & 3.0246 & 3.3804 \\
\hline & 0.95 & 1.9743 & 1.9869 & 2.0157 & 2.1026 & 2.3002 & 2.5758 & 2.8225 & 3.1988 & 3.4436 \\
\hline & 0.99 & 2.1775 & 2.1785 & 2.2478 & 2.3041 & 2.5136 & 2.8186 & 3.0644 & 3.5055 & 3.8360 \\
\hline \multirow[t]{4}{*}{$(2,2,4)$} & 0.75 & 1.2996 & 1.3130 & 1.3560 & 1.4631 & 1.6232 & 1.8317 & 2.1135 & 2.3161 & 2.5885 \\
\hline & 0.90 & 1.3673 & 1.3829 & 1.4296 & 1.5351 & 1.7296 & 1.9556 & 2.2134 & 2.5085 & 2.8036 \\
\hline & 0.95 & 1.4068 & 1.4228 & 1.4763 & 1.5822 & 1.7882 & 2.0168 & 2.2883 & 2.5934 & 2.8348 \\
\hline & 0.99 & 1.4786 & 1.4954 & 1.5546 & 1.6627 & 1.8607 & 2.1086 & 2.4006 & 2.7022 & 3.0201 \\
\hline \multirow[t]{4}{*}{$(2,3,4)$} & 0.75 & 1.2990 & 1.3085 & 1.3405 & 1.4176 & 1.5809 & 1.7873 & 2.0622 & 2.2633 & 2.5295 \\
\hline & 0.90 & 1.3667 & 1.3777 & 1.4153 & 1.4996 & 1.6593 & 1.9034 & 2.1558 & 2.4432 & 2.7307 \\
\hline & 0.95 & 1.4061 & 1.4175 & 1.4557 & 1.5426 & 1.7191 & 1.9294 & 2.2262 & 2.4694 & 2.7600 \\
\hline & 0.99 & 1.4777 & 1.4902 & 1.5275 & 1.6144 & 1.7883 & 2.0476 & 2.3443 & 2.6256 & 2.9345 \\
\hline
\end{tabular}

Table 4. Minimum median ratios to the specified life at producer's risk of 0.10

\begin{tabular}{|c|c|c|c|c|c|c|c|c|c|c|}
\hline \multirow[b]{2}{*}{$(a, b, \gamma)$} & \multirow[b]{2}{*}{$\mathrm{P} *$} & \multicolumn{7}{|c|}{$\eta$} & \multirow[b]{2}{*}{1.7} & \multirow[b]{2}{*}{1.9} \\
\hline & & 0.3 & 0.5 & 0.7 & 0.9 & 1.1 & 1.3 & 1.5 & & \\
\hline \multirow[t]{4}{*}{$(1,1,2)$} & 0.75 & 2.3909 & 2.5429 & 2.7027 & 2.9597 & 3.4091 & 3.5771 & 4.1274 & 4.2579 & 4.7588 \\
\hline & 0.90 & 2.9194 & 3.1138 & 3.3299 & 3.6160 & 3.9485 & 4.4820 & 4.9328 & 5.2686 & 5.8884 \\
\hline & 0.95 & 3.2919 & 3.4807 & 3.7015 & 4.1781 & 4.5723 & 5.0530 & 5.3843 & 5.7875 & 6.5506 \\
\hline & 0.99 & 3.9229 & 4.1101 & 4.3943 & 4.8300 & 5.3321 & 5.8442 & 6.6049 & 6.9882 & 7.4767 \\
\hline \multirow[t]{2}{*}{$(1,1,4)$} & 0.75 & 1.5234 & 1.5371 & 1.5950 & 1.7052 & 1.8873 & 2.0574 & 2.2912 & 2.5967 & 2.9022 \\
\hline & 0.90 & 1.6861 & 1.7026 & 1.7701 & 1.8588 & 2.0296 & 2.2886 & 2.5736 & 2.8200 & 3.1517 \\
\hline
\end{tabular}




\begin{tabular}{|c|c|c|c|c|c|c|c|c|c|c|}
\hline & 0.95 & 1.7825 & 1.7967 & 1.8459 & 2.0079 & 2.1615 & 2.3987 & 2.7202 & 2.9928 & 3.3449 \\
\hline & 0.99 & 1.9686 & 1.9698 & 2.0221 & 2.1803 & 2.3992 & 2.6358 & 2.9474 & 3.2615 & 3.6451 \\
\hline \multirow[t]{4}{*}{$(1,2,2)$} & 0.75 & 2.3569 & 2.4653 & 2.5793 & 2.8532 & 2.9882 & 3.3075 & 3.4845 & 3.9491 & 4.1225 \\
\hline & 0.90 & 2.8777 & 3.0308 & 3.1046 & 3.4292 & 3.6279 & 3.9348 & 4.2846 & 4.8558 & 5.1615 \\
\hline & 0.95 & 3.2196 & 3.3468 & 3.5059 & 3.7050 & 4.0532 & 4.4735 & 4.6967 & 5.3893 & 5.7509 \\
\hline & 0.99 & 3.9456 & 4.1464 & 4.1749 & 4.5076 & 4.8787 & 5.1730 & 5.6530 & 6.4773 & 6.7318 \\
\hline \multirow[t]{4}{*}{$(1,2,4)$} & 0.75 & 1.5224 & 1.5333 & 1.5685 & 1.6345 & 1.7546 & 1.9814 & 2.2095 & 2.5041 & 2.6459 \\
\hline & 0.90 & 1.6849 & 1.6975 & 1.7239 & 1.8075 & 1.9465 & 2.1971 & 2.3926 & 2.7116 & 3.0306 \\
\hline & 0.95 & 1.7833 & 1.7943 & 1.8194 & 1.8954 & 2.0706 & 2.3147 & 2.5352 & 2.8732 & 3.0904 \\
\hline & 0.99 & 1.9669 & 1.9678 & 2.0291 & 2.0788 & 2.2652 & 2.5376 & 2.7578 & 3.1536 & 3.4505 \\
\hline \multirow[t]{4}{*}{$(2,2,4)$} & 0.75 & 1.2349 & 1.2461 & 1.2825 & 1.3760 & 1.5184 & 1.7064 & 1.9689 & 2.1515 & 2.4046 \\
\hline & 0.90 & 1.2994 & 1.3129 & 1.3535 & 1.4470 & 1.6239 & 1.8313 & 2.0706 & 2.3466 & 2.6227 \\
\hline & 0.95 & 1.3368 & 1.3509 & 1.3982 & 1.4930 & 1.6817 & 1.8926 & 2.1457 & 2.4318 & 2.6547 \\
\hline & 0.99 & 1.4051 & 1.4201 & 1.4734 & 1.5711 & 1.7535 & 1.9836 & 2.2572 & 2.5395 & 2.8383 \\
\hline \multirow[t]{4}{*}{$(2,3,4)$} & 0.75 & 1.2345 & 1.2424 & 1.2693 & 1.3360 & 1.4829 & 1.6706 & 1.9276 & 2.1106 & 2.3589 \\
\hline & 0.90 & 1.2988 & 1.3083 & 1.3412 & 1.4159 & 1.5606 & 1.7869 & 2.0221 & 2.2918 & 2.5614 \\
\hline & 0.95 & 1.3362 & 1.3462 & 1.3800 & 1.4576 & 1.6193 & 1.8134 & 2.0924 & 2.3185 & 2.5912 \\
\hline & 0.99 & 1.4044 & 1.4155 & 1.4488 & 1.5273 & 1.6875 & 1.9298 & 2.2082 & 2.4723 & 2.7631 \\
\hline
\end{tabular}

\section{Selection Of Sampling Plan}

Tables 1 may be used for the selection of zero-one double sampling plan with truncated life test for the specified confidence level when the lifetime of the product follows Kumaraswamy-log-logistic distribution with different shape parameters.

Example.1 Assume that the lifetime of the product under consideration follows Kumaraswamy-log-logistic distribution with shape parameters $\mathrm{a}=1, \mathrm{~b}=2$ and $\gamma=4$. Suppose that the producer wants to establish that the true median life is greater than or equal to 2000 hours with the confidence level of 0.95 . The experimenter wants to stop the experiment at 1000 hours. This leads to the experiment termination multiplier, $\eta=0.5$. For the problem under consideration Table 1 gives a zero-one double sampling plan with the minimum sample sizes $\mathrm{n}_{1}=63$ and $\mathrm{n}_{2}=61$. This sampling plan is put into operation as follows

First, selected randomly 63 items from the lot and put on test for 1000 hours and accept the lot if no failure occurs and reject if more than one failure occurs during the experimental time. If one failure is observed select a second sample of size 61 and put on test for 1000 hours. Accept the lot if there are no failures in the second sample. otherwise, reject the lot.

The producer may be concerned with the acceptance probability as the quality improves because they want to minimize the producer's risk. Suppose that the producer is interested in knowing what quality level will lead to the producer's risk less than 0.05 . This can be answered from Table 3 . The minimum ratio for $a=1, b=2$ and $\gamma=4, \mathrm{P}^{*}=0.95$ and $\eta=0.5$ is 1.9869 . So, the true median required of the product should be at least 19869 hours.

Example.2 Suppose that the lifetime of the product follows a Kumaraswamy-log-logistic distribution with shape parameters $\mathrm{a}=2, \mathrm{~b}=3$ and $\gamma=4$. Assume that the producer wishes to establish that the true median life is larger than 1000 hours with the confidence level of 0.90 . The experimenter wants to terminate the experiment at 700 hours. For this problem Table 1 gives a zero-one double sampling plan with the minimum sample sizes $\mathrm{n}_{1}=31$ and $\mathrm{n}_{2}=30$. As the termination time leads to $\eta=0.7$. This sampling plan is carried out as follows

First, select 31 items from the lot and put on test for 700 hours and accept the lot if no failure occurs and reject the lot if more than one failure occurs during the experimental time. If one failure is observed select a second sample of size 30 and put on test for 700 hours. Accept the lot if there are no failures from the second sample and reject the lot otherwise.

The producer may be concerned with the acceptance probability as the quality improves because they want to minimize the producer's risk. Suppose that the producer is interested in knowing what quality level will 
lead to the producer's risk less than 0.10 . This can be answered from Table 4 . The minimum ratio for $a=2, b=3$ and $\gamma=4, P^{*}=0.90$ and $\eta=0.5$ is 1.3083 . So, the true median required of the product should be at least 13083 hours.

\section{Conclusion}

The double sampling plan with zero-one failure scheme is considered and tables are prepared to make decision of acceptance or rejection on the submitted lot under the assumption that the lifetime of the product following Kumaraswamy-log-logistic distribution with different shape parameters, which has wider applicability due to the inclusion of several sub-models.

A general double sampling plan would be preferable rather than a zero-one double sampling plan. Therefore, a general double sampling plan for truncated life test is under preparation as a future work.

\section{References}

[1] M. Aslam, and C.H. Jun, A double acceptance sampling plan for generalized log-logistic distributions with known shape parameters, Journal of Applied Statistics, 37(3), 2010, 405-414.

[2] G. M. Cordeiro, and M. de Castro, A new family of generalized distributions, Journal of Statistical Computation and Simulation, 81, 2011, 883-898.

[3] A.J. Duncan, Quality Control and Industrial Statistics, 5th ed., Richard D. Irwin, Homewood, Illinois, 1986.

[4] B. Epstein, Truncated life tests in the exponential case, Ann. Math. Statist. 25, 1954, 555-564.

[5] H.P. Goode, and J.H.K. Kao, Sampling plans based on the Weibull distribution, In Proceeding of the Seventh National Symposium on Reliability and Quality Control, Philadelphia, 1961, 24-40.

[6] S.S. Gupta, Life test sampling plans for normal and lognormal distributions, Technometrics 4, 1962, $151-175$.

[7] S.S. Gupta, and P.A. Groll, Gamma distribution in acceptance sampling based on life tests, J.Am. Statist. Assoc. 56, 1961, 942-970.

[8] P. Kumaraswamy, A generalized probability density function for double-bounded random processes, Journal of Hydrology 46, 1980, 79-88. 